\title{
A Fractional Variable Partial Update Least Mean Square Algorithm (FVPULMS) for Communication Channel Estimation
}

\author{
S. A. Akinboro ${ }^{1 *}$, A. Omotosho ${ }^{2}$, E. A. Oluwatosin ${ }^{1}$ \\ ${ }^{1}$ Department of Computer Science and Information Technology, Bells University of Technology, Ota, Nigeria. \\ ${ }^{2}$ Department of Computer Science, Landmark University, Omu Aran, Nigeria.
}

\begin{abstract}
The study presents a study on communication channel estimation algorithms. Fractional Variable Partial Update Least Mean Square algorithm (FVPULMS) is proposed. The model consists of input signal, unknown channel, an adaptive filter and an adaptation algorithm. The adaptive filter is a finite impulse response transversal adaptive filter. The adaptation algorithm is a FVPULMS algorithm and the filter update uses coefficient with index factors of three and five. The proposed algorithm was compared with Variable Partial Update Least Mean Square (VPULMS) and Full Update Least Mean Square (FULMS) algorithms. The simulation was carried out using fixed step sizes and variable step sizes of $(<$ and $>$ ). The result showed that FVPULMS algorithm has enhanced average performance efficiency in terms of estimated signal error reduction at the receiver station compared with VPULMS and FULMS algorithms, and can help to achieve improved signal error at the receiver station.
\end{abstract}

KEYWORDS: Adaptation, Channel, step size, convergence speed, computational complexity, error state.

[Received September 22, 2017; Revised April 07, 2018; Accepted May 01, 2018]

Print ISSN: 0189-9546 | Online ISSN: 2437-2110

\section{INTRODUCTION}

A communication channel is referred to as either a physical transmission medium or a logical connection over a multiplexed medium. Communication channel is used to convey an information signal, from one or several senders to one or several receivers. The rapid growth of technology in recent decades has changed the whole dimension of communications. Communications systems development is increasing considerably and there are more issues of additive noise, signal interference and echo. These issues result to errors in data transmission. Adaptive filtering can be used to equalize any linear distortions on the communication channel by processing the signal at the receiver station.

Statistical methods are usually used to develop algorithms that can be used to process transmitted signals. This is done by filtering the signals to predict and estimate the desired signal thus preventing data loss as a result of signal errors. According to estimation theory, it is assumed that the desired signal is hidden in the noisy signal. The noise adds inaccuracy to the signal and the need for an accurate signal calls for adequate signal processing scheme.

The retrieval of information about a particular channel whether from a sent signal or a received signal is referred to as Channel Estimation (Akinboro et al. 2011). The two general approaches to channel estimations are the Least Mean Square algorithm (LMS) and the Fractional Least Mean Square (FLMS) algorithm. LMS algorithm has been widely deployed due to its simplicity and robustness (Arenas-Garcia et al., 2005). Its convergence rate is very slow and stability of error is not so efficient. LMS has a very high computational complexity hence a modified LMS has been developed with a variable step-size called Variable Least Mean Square algorithm (VLMS).

The computational complexity of the VLMS is low compared to the original LMS. Its convergence speed and steady-state of error is also more efficient compared to LMS with constant step-size. Most Adaptive algorithms take a lot of iterations to give satisfactory result when the number of input elements is too large because of their slow initial convergence. The step size is used to compute the Mean Square Error (MSE). VLMS algorithm can be used in adaptive beam forming systems to steer the beam of the resulting antenna or in any adaptive signal processing system. It can also be used for weight adjustment of any antenna or a system where the weights have to be found very fast.

The FLMS algorithm, is based on fractional order calculus was proposed with a faster convergence and a more stable error state (Dubey \& Rout 2012). The Fractional Least Mean Square algorithm (FLMS) has a higher computational complexity and faster convergence. In Akinboro et al. (2011), a Variable Partial Update Least Mean Square (VPULMS) algorithm with low computational complexity, considerable convergence speed and improved memory load was proposed. Thus, combining the benefits of VPULMS and FLMS algorithms into what we called Fractional Variable Partial Update Least Mean Square algorithm (FVPULMS) will help to develop a more efficient, effective and reliable algorithm with faster convergence and a more stable error state.

\section{LITERATURE REVIEW}

Several research works have been done to develop different variations of LMS algorithms. Maity et al. (2012) proposed a modified form of LMS algorithm called "Fast 
Adaptive Least Mean Square" algorithm. The algorithm has an adaptive step size that gives a fast convergence irrespective of the number of input elements $(\mathrm{N})$ with a typical limiting error of 10-10. The results of the simulations showed that the new algorithm has a very fast convergence irrespective of the number of input elements. Kaur et al. (2012) presented an analysis on the performance of tracking speed and stability of the adaptive gradient filtering algorithms. According to the simulation results of the noise cancellation, the algorithm could get stabilized only after 20 iterative operations. It can provide stronger ability to boost the Signal to Noise Ratio (SNR) of weak signal compared to LMS, Normalized LMS, and the Variable Step-size LMS filters.

An intuitive way to improve the performance of the LMS algorithm is to make the step size variable rather than fixed. This is done by chosing large step size values during the initial convergence of the LMS algorithm, and use small step size values when the system is close to its steady state. Ajjaiah et al., (2012) introduced a new concept to vary the step size based on evolutionary programming algorithm. The results generated by this method are robust and does not require any pre-setting of involved parameters. Jamel (2013) presented a new method for variable step size LMS (VLMS) algorithm. The proposed algorithm is based on an absolute mean of estimation, current and prior error vector. The main purpose of this algorithm is to enhance the performance of adaptive echo cancellation system. The algorithm was tested with real speech input signal and the result shows that it has fast convergence time, low level of mis-adjustment, and high Echo Return Loss Enhancement (ERLE).

The amount of ERLE using proposed algorithm compared with LMS and VLMS algorithms is about $10 \mathrm{~dB}$ and $8 \mathrm{~dB}$ respectively. Singh et al. (2013) implemented and made comparison of adaptive algorithm, called Complementary Pair Variable Step Size Least Mean Square algorithm (CPVLMS). Simulation experiments were done in LabVIEW to compare the learning curves of the CPVLMS and CPLMS algorithm. The results showed that the CPVLMS algorithm provides faster convergence speed and small steady state error than the CPLMS algorithm. Akinboro et al. (2011) proposed a Variable Partial Update model for adaptive communication channel estimation to improve on signal error at receiver's station.

The proposed model consists of an adaptive finite impulse response filter and Variable Partial Update LMS (VPULMS) adaptation algorithm. The performance of the proposed model was simulated in comparison with the full update model and the result of the evaluation indicated that in terms of computational complexity, memory load and convergence rate, the proposed model performed better than the full update model.

An Identification of input non-linear control autoregressive systems using fractional signal processing approach was presented by Chaudhary et al. (2013). The design scheme consists of parameterization of Input Nonlinear Control Autoregressive (INCAR) systems, to obtain linear-in-parameter models. Fractional LMS (FLMS) algorithm was used for the unknown parameter vectors. The performance of the algorithm was carried out with third-order volterra LMS and kernel LMS (KLMS) algorithms based on the convergence to the true values of INCAR systems. The results demonstrated that the FLMS algorithm provided a better accuracy and convergence compared to those of volterra LMS and KLMS under different scenarios using lowto-high signal-to-noise ratio.

Similarly, Shoaib and Qureshi (2014) proposed a modification of the FLMS algorithm to provide a better achievement in the performance of the filter. The algorithm was able to handle non-linear problems with less computational burden by avoiding the evaluation of complex gamma function. It includes the incorporation of an adjustable gain parameter in the weight adaptation equation of the original FLMS algorithm and absorbs the gamma function in the fractional step size parameter. Modified FLMS was compared with LMS and KLMS. The simulation results for the time series with and without noise confirmed that the Modified FLMS has an improved prediction capability compared to LMS and KLMS.

Raja and Chaudhary (2014) presented a novel application of fractional adaptive algorithms for parameter identification of Box-Jenkins (BJ) systems; "Adaptive strategies for parameter estimation of Box-Jenkins systems". They adapted an unknown parameter vector of the BJ system using the fractional least mean square (FLMS) algorithm for three different values of the fractional order. The estimated result was compared with the state of the art volterra LMS and KLMS adaptive algorithms. The reliability and effectiveness of the algorithm were analyzed through the results of the statistical analysis. Based on sufficient large number of independent runs, the FLMS algorithm compared to the other algorithms provided consistent accuracy and convergence for the Box-Jenkins systems in different scenarios.

In summary, combining the benefits of VPULMS and FLMS algorithms can result in a more efficient, effective and reliable algorithm with a better computation, faster convergence and a more stable error state.

\section{ARCHITECTURE OF PROPOSED MODEL}

The model in Figure 1 comprises of four specific modules; an unknown channel, finite impulse response (FIR) filter, adaption algorithm and a summer $\left(\sum\right)$. The unknown channel is placed parallel to the finite impulse response (FIR) filter so that the input signal $x(n)$ can be transmitted simultaneously. The estimation is parallel and adaptive and this is so because in wireless situation, the paths followed by the signal between the transmitter and the receiver may keep changing.

The signal $d(n)$, transmitted through the unknown channel is the desired training signal while the signal $y(n)$ transmitted through the FIR filter is the estimated training signal. The two signals are transmitted through the summer $\left(\sum\right)$ to realize the estimated error signal $\mathrm{e}(\mathrm{n})$. The estimated error signal is then used to update the coefficient of the FIR 
filter using the adaptation algorithm called FVPULMS. The adaptation algorithm uses index factors of three and five to update the coefficients of the filter (Akinboro et al. 2011).

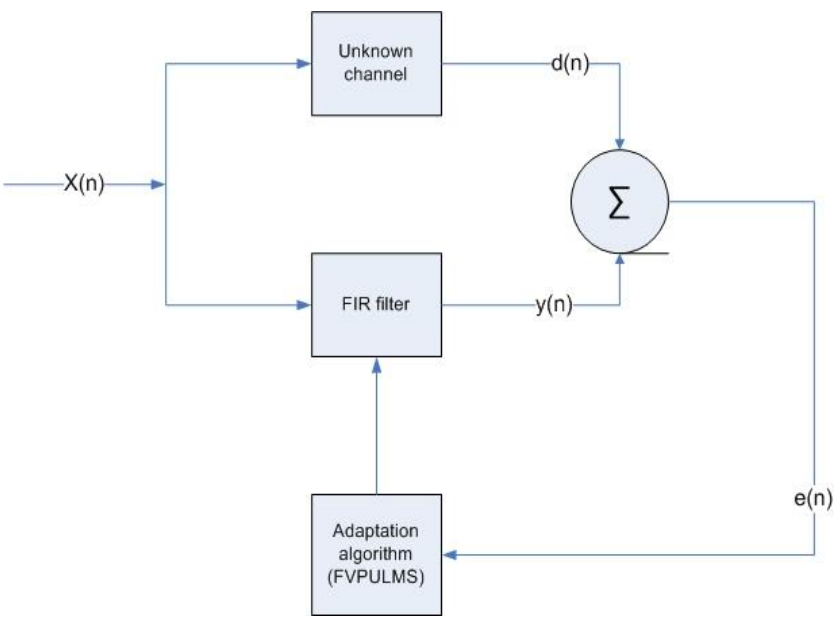

Figure 1: Block Diagram for FVPULMS Model.

\section{A. Algorithm for the Proposed FVPULMS}

It is assumed that the FVPULMS filter in Figure 2 is a standard transversal FIR filter of length $L \geq 5$. Let ( ) be the input sequence and let $(w)$ denote the coefficient of the adaptive filter.
The adaptive weight update for the $K^{\text {th }}$ element is given as follows;

$w_{k(n+1)}=w_{k(n-1), j}-\mu_{1}\left[\frac{2 e(n)}{2 w k}\right]-\mu_{2}\left[\frac{2 e(n)}{2 w k}\right]$

where $\mu_{1}$ and $\mu_{2}$ are fixed step sizes.

Step 2: Compute the estimated Error

$$
\begin{aligned}
& \mathrm{e}(\mathrm{n})=[d(n)]^{2}+[y(n)]^{2}-2[d(n) \times y(n) \times \\
& \mathrm{e}(\mathrm{n})=\left[\begin{array}{lll}
d(n)]^{2}+ & {\left[\sum_{i=1}^{n-1} w_{i(n), j} \times x_{(n-i), j}\right.}
\end{array}\right]^{2}- \\
& 2 d(n) \sum_{i=0}^{n-1} w_{i(n), j} \times x_{(n-i), j}
\end{aligned}
$$

Step 3: Update the Coefficient of the Adaptive Filter This can be carried out by taking the fractional derivative of $\mathrm{v}$-order. $\mathrm{v}$ is a real number between 0 and 1 (we assume $\mathrm{v}=$ $0.5)$.

$$
\begin{gathered}
{\left[\partial / \partial_{w k}\right]^{v} e(n)=2 \sum_{i=0}^{n-1} w_{i(n), j} \times x_{(n-i), j} \times} \\
\sum_{i=0}^{n-1}\left[\left(\partial / \partial_{w k}\right)^{v} w_{i(n), j}\right] \times x_{(n-i), j}-2 \mathrm{~d}(\mathrm{n}) \times \\
\sum_{i=0}^{n-1}\left[\left(\partial / \partial_{w k}\right)^{v} w_{i(n), j}\right] \times x_{(n-i), j}
\end{gathered}
$$

After simplifying eqn (5), we get

$$
\left[\partial / \partial_{w k}\right]^{v} e(n)=-2 e(n) x_{(n-i), j} D^{v} w_{k(n)}
$$

where $D^{v}$ is equal to Riemann Liouvilli differential operator.

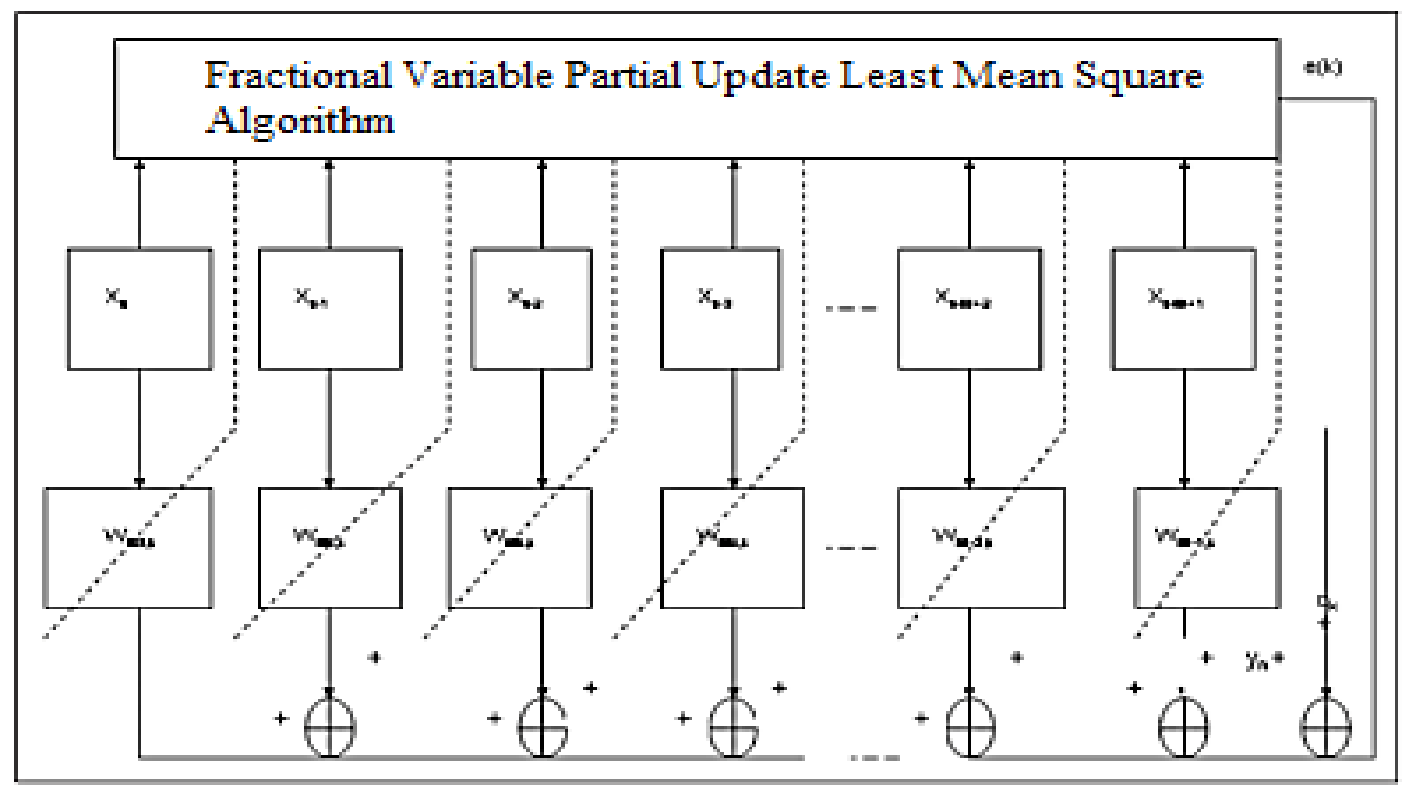

Figure 2: The Transversal FIR Filter Structure for the FVPULMS Model adopted from (Akinboro et al. 2011).

Step 1: Compute the output of the $K^{\text {th }}$ element of the Adaptive Filter

$$
y(n)=w_{k(n), j}^{T} \times x_{n, j}
$$

where $\mathrm{j} \in \mathrm{S}, \mathrm{T}=$ transpose.

$S=\{w 3, w 5, w 6 \ldots \ldots \ldots \ldots\}$, weights with index factors of three (3) and five (5) used for the update of the filter.
Further substation for $D^{v}$ gives:

$$
\left[\partial / \partial_{w k}\right]^{v} e(n)=-2 e(n) x_{(n-i), j}\left[\frac{1}{\Gamma(2-v)}\right] w_{k^{1-v}(n)}
$$

We substitute (7) into eqn (2). Therefore:

$$
\begin{array}{r}
w_{k(n-1)}=w_{k(n-1), j}+2 \mu_{1} e(n) x_{(n-i), j}+ \\
2 \mu_{2} e(n) x_{(n-i), j}\left[\frac{1}{\Gamma(2-v)} w_{k^{1-v}(n)}\right]
\end{array}
$$


assuming that $\mu_{-} 1=\mu \_2$

Step 4: Update the Variable Step-Size of the adaptive Filter $\mu(n+1)=\alpha \mu(n-1)+\mu(n) p(n)$

$$
\begin{aligned}
& \mu(n+1)=\left\{\begin{array}{cc}
\mu_{\max } & \text { if } j \mu(n+1)>\mu_{\max }, \\
\mu_{\min } & \text { if } j \mu(n+1)<\mu_{\min }, \\
\mu(n+1) & \text { otherwise }
\end{array}\right. \\
& \gamma(n)=\left\{\begin{array}{cc}
\beta \gamma(n-1) & \text { if } j \mu(n+1)>\mu_{\max } \\
\gamma(n-1) & \text { otherwise }
\end{array}\right.
\end{aligned}
$$

where $\gamma$ and $\beta$ are constant values, $0<\gamma, \beta>1$. The algorithm will adjust the parameter $\gamma$ with the constant $\beta$. To ensure convergence the parameter $\beta$ must satisfy that $0<$ $\beta<1$.

\section{RESULTS AND DISCUSSION}

Simulation program was developed for the proposed Fractional Variable Partial Update Least Mean Square algorithm (FVPULMS), Variable Partial Update Least Mean Square (VPULMS) and Full Update Least Mean Square (FULMS) algorithms using MATLAB 7.0. Simulation parameters include pseudo random number generator with zero means, variance of one as input discrete signal sequence, and introduction of noise signal sequence of 0.8 noise level. These signals form the desired signals that were input to the FIR filter. The outputs from the filter form the actual signal which was subtracted from the desired signal to obtain the mean square error. Simulation was performed using fifty

Table 1: Simulation Parameter for Fixed Step Size $(\mu)$.

\begin{tabular}{ccccccc}
\hline $\begin{array}{c}\text { Step Size } \\
\text { Value }\end{array}$ & $\boldsymbol{\mu}_{\max }$ & $\boldsymbol{\mu}_{\min }$ & Gamma & Alpha & Beta & Length \\
\hline 0.001 & 1.9 & 0.001 & 0.007 & 0.2 & 0.01 & 50 \\
0.0011 & 1.9 & 0.001 & 0.007 & 0.2 & 0.01 & 50 \\
0.0013 & 1.9 & 0.001 & 0.007 & 0.2 & 0.01 & 50 \\
0.0017 & 1.9 & 0.001 & 0.007 & 0.2 & 0.01 & 50 \\
0.0020 & 1.9 & 0.001 & 0.007 & 0.2 & 0.01 & 50 \\
0.0030 & 1.9 & 0.001 & 0.007 & 0.2 & 0.01 & 50 \\
\hline
\end{tabular}

Table 2 Efficiencies of FULMS, VPULMS, FVPULMS for Fixed Step Size.

\begin{tabular}{cccc}
\hline Step Size & FULMS \% & VPULMS \% & FVPULMS \% \\
\hline 0.001 & 72 & 86 & 94 \\
0.0011 & 70 & 80 & 98 \\
0.0013 & 74 & 82 & 90 \\
0.0017 & 70 & 86 & 94 \\
0.0020 & 76 & 84 & 96 \\
0.0030 & 78 & 80 & 92
\end{tabular}

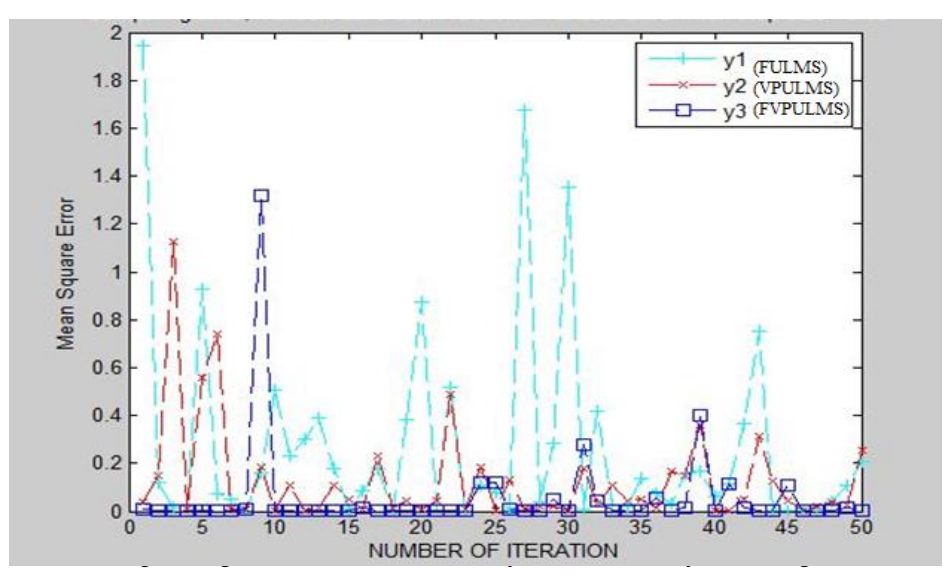

Figure 3: Mean Square Error comparison for Step Size of 0.001. different sets of input data with different values of fixed and variable step size parameters to obtain the average result of the mean square error.

Table 1 shows the values of the fixed step size and other simulation parameters. FULMS, VPULMS and FVPULMS were simulated using various step sizes. Figure 3 shows the comparison results for algorithms with (0.001) step size and Table 2 shows the efficiencies of the algorithms with different fixed step sizes.

The algorithms was also tested with variable step size for the case when the step size is less than the minimum step size $(\mu<\mu$ min $)$ as shown in Table 3 . Figure 4 shows the comparison results for algorithms with (0.0036) step size and Table 4 shows the efficiencies of the algorithms with different variable step sizes. Finally we considered the case when the step size is larger than maximum step size $(\mu>\mu \max )$. Table 5 shows the parameters used for the simulation while Figure 5 shows the comparison for algorithms with (0.25) step size and Table 6 shows the efficiencies of the algorithms with different variable step sizes.

Table 3 Mean Square Comparison Using Variable Step Size, for $\mu<\mu_{\min }$.

\begin{tabular}{ccccccc}
\hline $\begin{array}{c}\text { Step } \\
\text { Size } \\
\text { Value }\end{array}$ & $\boldsymbol{\mu}_{\max }$ & $\boldsymbol{\mu}_{\min }$ & Gamma & Alpha & Beta & Length \\
\hline 0.0036 & 1.9 & 0.001 & 0.007 & 0.2 & 0.01 & 50 \\
0.049 & 1.9 & 0.001 & 0.007 & 0.2 & 0.01 & 50 \\
0.052 & 1.9 & 0.001 & 0.007 & 0.2 & 0.01 & 50 \\
\hline
\end{tabular}

Table 4 Efficiencies of FULMS, VPULMS, FVPULMS for Variable Step Size $\mu<\mu_{\min }$

\begin{tabular}{cccc}
\hline Step Size & FULMS \% & VPULMS\% & FVPULMS \% \\
\hline 0.0036 & 64 & 78 & 84 \\
0.0049 & 68 & 74 & 92 \\
0.0052 & 66 & 72 & 84 \\
\hline
\end{tabular}

Table 5 Mean Square Comparison Using Variable Step Size, for $\mu>\mu_{\max }$.

\begin{tabular}{ccccccc}
\hline $\begin{array}{c}\text { Step } \\
\text { Size } \\
\text { Value }\end{array}$ & $\boldsymbol{\mu}_{\max }$ & $\boldsymbol{\mu}_{\min }$ & Gamma & Alpha & Beta & Length \\
\hline 0.25 & 1.9 & 0.001 & 0.007 & 0.2 & 0.01 & 50 \\
0.30 & 1.9 & 0.001 & 0.007 & 0.2 & 0.01 & 50 \\
0.40 & 1.9 & 0.001 & 0.007 & 0.2 & 0.01 & 50 \\
\hline
\end{tabular}

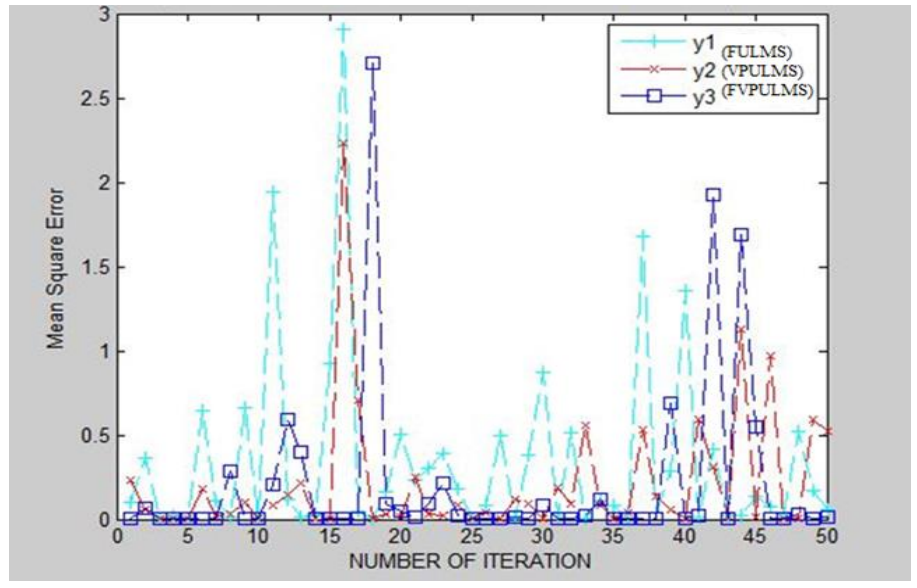

Figure 4: Mean Square Error comparison for Step Size of 0.0036 . 


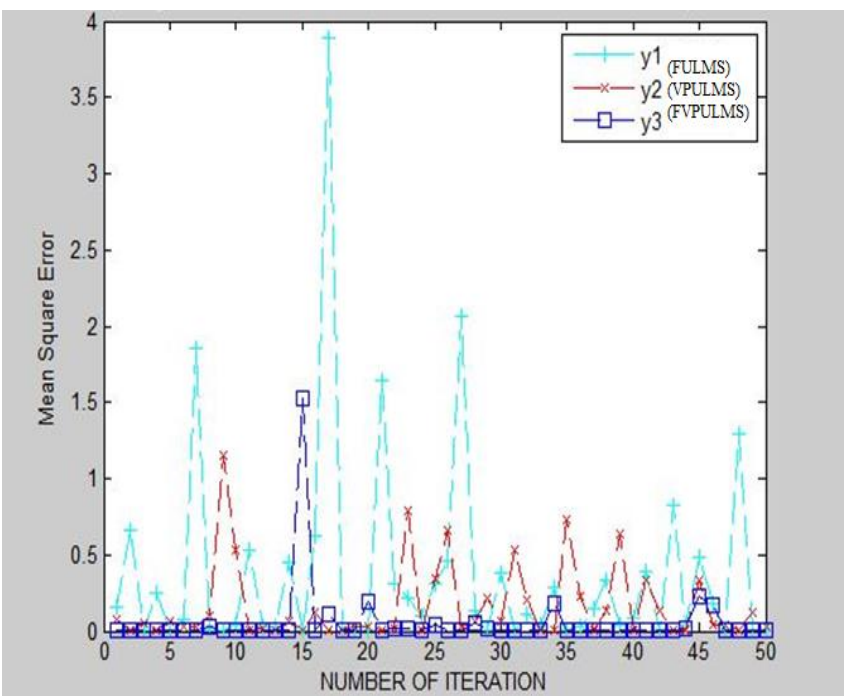

Figure 5: Mean Square Error comparison for Step Size of 0.25.

Table 6 Efficiencies of FULMS, VPULMS, FVPULMS for Variable Step Size $\mu>\mu_{\max }$.

\begin{tabular}{cccc}
\hline Step Size & FULMS \% & VPULMS \% & FVPULMS\% \\
\hline 0.25 & 62 & 80 & 98 \\
0.30 & 68 & 76 & 92 \\
0.40 & 64 & 74 & 90 \\
\hline
\end{tabular}

\section{$\mathrm{V}$ CONCLUSION}

A model for communication channel estimation called FVPULMS algorithm was proposed, which combines the goodness of FLMS and VPULMS. The proposed model implemented adaptive filter that is finite impulse response adaptive filter. Fixed and variable step sizes were used as major parameters among others to generate mean error and mean square error. The efficiency of the algorithms (FULMS, VPULMS, FVPULMS) were determined by comparing the mean square errors of the different algorithms. The update of the filter coefficient was carried out using index factors of three and five. The simulation results showed that FVPULMS has a better performance compared to FULMS and VPULMS algorithms. Therefore, the proposed model can lead to a more efficient, effective and reliable quality transmission. Also FVPULMS is capable of resulting in stable error state and faster convergence rate of the transmitted signal.

\section{REFERENCES}

Sengupta, C. (1998). Algorithms and architectures for channel estimation in wireless CDMA communication systems (Doctoral dissertation).

Arenas-Garcia, J.; V., Gomez-Verdejo, \& A. R., Figueiras-Vidal, (2005). New algorithms for improved adaptive convex combination of LMS transversal filters. IEEE Transactions on Instrumentation and measurement, 54(6): 2239-2249.

Dubey, S. K., \& Rout, N. K. (2012). FLMS algorithm for acoustic echo cancellation and its comparison with LMS. In 1st IEEE International Conference on Recent Advances in Information Technology (RAIT), Dhanbad, India, 852-856.

Akinboro, S.A.; G.A, Aderounmu, E.A, Olajubu, A.O, Ajayi, I.K., Ogundoyin \& O.M., Olaniyan, (2011). An Improved Computational Model for Adaptive Communication Channel Estimation. Int. J. of Computers, Communications \& Control, 4(2), 204-213.

Maity, S, Dasgupta, S, \& Gupta, B. (2012). Fast Adaptive Least Mean Square Algorithm. PIERS Proceedings, Kuala Lumpur, MALAYSIA, March 27 - 30.

Ajjaiah, H.B.M.; P. V., Hunagund, M. K., Singh, \& P. V., Rao. (2012). Adaptive Variable Step Size in LMS Algorithm Using Evolutionary Programming: VSSLMSEV. Signal Processing: An International Journal (SPIJ), 6(2): 78.

Jamel, T. M. (2013). Performance enhancement of adaptive acoustic echo canceller using a new time varying step size LMS algorithm (NVSSLMS). International Journal of Advancements in Computing Technology (IJACT), Korea, 3(1): 1-10.

Singh, G.; M., Kaura, K.V.P, Singha, \& R., Kaurb. (2013). Performance Analysis of CPVSS-LMS and CP-LMS adaptive algorithms in LabVIEW. International Journal of Current Engineering and Technology, 3(4): 1238 - 1241.

Chaudhary, N. I.; M. A. Z., Raja, J. A., Khan, \& M. S., Aslam. (2013). Identification of input nonlinear control autoregressive systems using fractional signal processing approach. The Scientific World Journal, 2013(1): 1 - 13

Shoaib, B., \& Qureshi, I. M. (2014). A modified fractional least mean square algorithm for chaotic and nonstationary time series prediction. Chinese Physics B, 23(3): $1-6$.

Raja, M. A. Z., \& Chaudhary, N. I. (2014). Adaptive strategies for parameter estimation of Box-Jenkins systems. IET Signal Processing, 8(9): 968-980. 